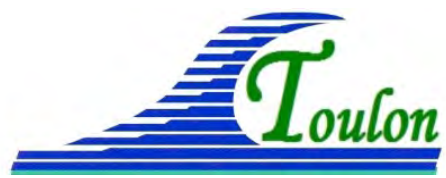

XIV èmes Journées Nationales Génie Côtier - Génie Civil Toulon, 29 juin au $1^{\text {er }}$ juillet 2016

DOI:10.5150/jngcgc.2016.048 (c) Editions Paralia CFL

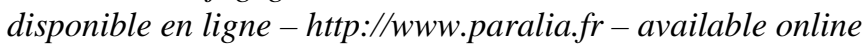

\title{
Maitrise de la vulnérabilité des ouvrages maritimes et vérification de la capacité portante sous bruit de fond
}

\author{
Maxime FONTAN $^{1}$, Thierry VASSAIL $^{1}$, Pierre Eric THEVENIN $^{1}$
}

1. OXAND, 49 Avenue Franklin Roosevelt, 77210 Avon, France.

maxime.fontan@oxand.com ; thierry.vassail@oxand.com ; pe.thevenin@oxand.com

\section{Résumé :}

Les ouvrages du génie civil en béton armé situés en milieu marin ou fluvial sont exposés à des actions environnementales sévères caractérisées par des attaques physiques et chimiques particulières (vagues, ions chlorure, corrosion, etc.). Les ouvrages maritimes, notamment en béton armé, sont particulièrement sensibles à ces actions et leur durabilité peut être impactée. Ces actions, par leurs conséquences, peuvent augmenter la vulnérabilité et diminuer la disponibilité des ouvrages en phase d'exploitation. Ces deux indicateurs représentent un coût non négligeable pour les gestionnaires si aucune action de maintenance ni prévention n’est réalisée.

Afin d'accompagner les gestionnaires d'ouvrage dans la maitrise de la vulnérabilité et de la disponibilité de leur patrimoine, OXAND propose la solution d'audit structural $\mathrm{SIMEO}^{\mathrm{TM}} 4 \mathrm{D}$ basée sur la capture du comportement dynamique des ouvrages. Cette solution, développée depuis plus de 15 années, permet à l'aide d'une instrumentation légère et portative de réaliser un diagnostic de structure à un rendement élevée (de l'ordre du kilomètre par jour dans le cadre d'une digue). L'exploitation des résultats permet non seulement d'identifier et de localiser de manière non-destructive et sans arrêt d'exploitation les zones de l'ouvrage présentant un comportement singulier, donc mettant en évidence un désordre ou une pathologie passés ou en cours de développement. Cette solution d'audit peut également être employée pour vérifier la capacité portante d'un ouvrage en mesurant sa réponse dynamique réelle sous chargement contrôlé et en la comparant aux valeurs attendues.

Cet article introduit les principes physiques de la solution SIMEO ${ }^{\mathrm{TM}} 4 \mathrm{D}$ illustrés par différentes applications mettant en avant les gains apportés pour les gestionnaires d'ouvrages et en particulier pour le compte de l'Etablissement du Service d'Infrastructure de la Défense de Toulon.

Mots-clés : Vulnérabilité des ouvrages, $\mathrm{SIMEO}^{\mathrm{TM}} 4 \mathrm{D}$, Capacité de chargement, Béton armé, Bruit de fond, Caractéristiques dynamiques, Fréquence propre, Déformée modale, Optimisation.

\section{Introduction}

La maintenance des ouvrages maritimes est devenue un enjeu majeur pour les gestionnaires qui doivent concilier des niveaux élevés de sécurité et de disponibilité de 


\section{Thème 4 - Ouvrages portuaires, offshore et de plaisance}

leurs installations couplés à une tendance à la baisse des budgets de maintenance. Compte tenu du vieillissement des ouvrages en béton armé situés en milieu agressif tel que les côtes ou fleuves, maintenir dans le meilleur état possible un patrimoine immobilier nécessite au préalable d’identifier son état structural général. La cartographie de cet état est souvent problématique dans le cas des ouvrages anciens dont la connaissance des actions appliquées (surcharges éventuelles au cours de la vie de l'ouvrage, renforcements et adaptations effectués) est souvent partielle et la documentation (notes de calcul, plans d'exécution, etc.) perdue ou incomplète. L'utilisation d'une méthode rapide d'intervention, et sans arrêt d'exploitation, qui apporterait des éléments quantitatifs et qualitatifs permettant de cartographier à haut rendement un ou plusieurs ouvrages serait alors un outil précieux pour améliorer la connaissance de l'état structural du patrimoine. L'analyse de la réponse dynamique de structures sollicitées par le bruit de fond ambiant est une solution qui a démontré, au cours des 15 dernières années, sa capacité à caractériser leur état structural. Le tableau 1 illustre différentes applications réalisées sur différents types d'ouvrages pour différents besoins (BOUTIN \& HANS, 2009 ; DUNAND, 2005).

La capacité à cartographier rapidement l'état structural sans connaissance préalable et la vérification de la capacité portante sont des applications particulièrement utiles pour les gestionnaires d'ouvrages maritimes ou fluviaux souhaitant maitriser la sécurité et la disponibilité de leurs installations. Ces deux points sont abordés dans le cadre d’un projet réalisé pour le compte de l'Etablissement du Service d'Infrastructure de la Défense (ESID) de Toulon, après avoir introduit les principes de l'audit structural sous bruit de fond développé par OXAND via sa solution $\mathrm{SIMEO}^{\mathrm{TM}} 4 \mathrm{D}$.

\section{L'audit structural sous bruit de fond}

\subsection{Principes fondamentaux du bruit de fond}

Les activités naturelles et humaines génèrent constamment des ondes qui se déplacent à travers le sol et les ouvrages. On peut citer par exemple le passage d'un véhicule dont les ondes vont solliciter les bâtiments urbains ou encore le vent qui va solliciter les structures d'un quai par exemple. L'ensemble de ces sollicitations constitue le bruit de fond, ou bruit ambiant, qui peut être assimilée à un bruit blanc dont la principale caractéristique est d'imposer un signal d'excitation ayant une puissance identique quelle que soit la fréquence étudiée. Ainsi, lors de la réalisation d'un audit, le bruit de fond va solliciter la structure étudiée avec la même énergie sur l'ensemble du spectre des fréquences. L'analyse de la réponse dynamique d'une structure soumise à cette excitation permet de mettre en évidence les variations spatiales ou temporelles des caractéristiques dynamiques de l'ouvrage et d'identifier la présence d'un désordre ou d'un comportement singulier. Le (Tableau 1) illustre différents cas d'application de la méthode SIMEO ${ }^{\mathrm{TM}} 4 \mathrm{D}$. 


\section{XIV èmes Journées Nationales Génie Côtier - Génie Civil \\ Toulon, 29 juin au $1^{\text {er }}$ juillet 2016}

Tableau 1. Différents cas d'application de l'étude du comportement dynamique des ouvrages sous bruit de fond.

\begin{tabular}{|c|c|c|}
\hline Besoin & Ouvrage étudié & Gains apportés \\
\hline $\begin{array}{l}\text { Vérification de } \\
\text { la capacité } \\
\text { portante }\end{array}$ & $\begin{array}{l}\text { Appontements, } \\
\text { quais, viaducs }\end{array}$ & $\begin{array}{l}\text { - Augmentation du niveau de capacité portante } \\
\text { - Optimisation de la disponibilité des installations }\end{array}$ \\
\hline $\begin{array}{l}\text { Connaissance } \\
\text { structurale }\end{array}$ & $\begin{array}{l}\text { Ouvrages d'art } \\
\text { type viaduc, } \\
\text { appontement }\end{array}$ & $\begin{array}{l}\text { - Identification et localisation des zones singulières } \\
\text { - Définition d'une signature mécanique de référence } \\
\text { - Priorisation et hiérarchisation des interventions } \\
\text { - Caractérisation de l'état d'endommagement à partir } \\
\text { d'indicateurs d'état structural }\end{array}$ \\
\hline $\begin{array}{l}\text { Suivi } \\
\text { périodique }\end{array}$ & $\begin{array}{l}\text { Liaisons mât- } \\
\text { fondation des } \\
\text { éoliennes }\end{array}$ & $\begin{array}{l}\text { - Maintien des structures en état de fonctionnement } \\
\text { - Anticipation des risques de ruine }\end{array}$ \\
\hline $\begin{array}{l}\text { Audit de } \\
\text { vulnérabilité } \\
\text { sismique }\end{array}$ & $\begin{array}{l}\text { Bâtiments de } \\
\text { grande hauteur }\end{array}$ & $\begin{array}{l}\text { - Vérification de la sécurité des personnes en cas de } \\
\text { séisme } \\
\text { - Identification des sources de dommage liées aux } \\
\text { équipements }\end{array}$ \\
\hline
\end{tabular}

\subsection{Etat structural réel et caractéristiques dynamiques}

Les caractéristiques dynamiques d'un ouvrage sont directement fonction de sa raideur et de sa masse, voir équation 1.

$$
f_{i}=\frac{i^{2}}{2 \pi} \sqrt{\frac{K}{M}}
$$

où : $f_{i}$ est la $i^{\text {ème }}$ fréquence propre $f$ de la structure étudiée, $K$ la matrice de raideur et $M$ la matrice de masse de l'ouvrage.

La masse d'un ouvrage ne varie pas durant la vie d'un ouvrage, à l'exception d'une modification d'usage ou d'un accident important dont les conséquences seraient visuellement observables. En revanche, la mesure de la raideur d'un ouvrage est plus complexe car elle combine plusieurs paramètres tels que :

- la nature des matériaux utilisés et leur état de dégradation ;

- la géométrie des éléments constitutifs ;

- la nature des liaisons entre ces éléments ;

- les conditions aux limites.

La valeur de la raideur est un indicateur important de l'état structural et son évolution est un paramètre significatif de l'évolution de cet état au cours du temps. Sans modification de la masse, la mesure de la fréquence propre est une mesure indirecte de la raideur et de l'état structural de l'ouvrage étudié. En effet, THILLARD et al. (2015) ont montré, à partir des données issues du benchmark SMART2013 (RICHARD et al., 2016) que l'estimation du niveau de dégradation peut être mesurée à travers l'évolution 
de la fréquence propre pour un mode particulier. La figure 1 illustre l'évolution de la chute de fréquence du premier mode en fonction de l'application d'un signal sismique endommageant progressivement l'ouvrage (maquette à l'échelle 1/3 d'un bâtiment $\mathrm{R}+2$ ). L'analyse des résultats a permis de confirmer que la chute de fréquence propre est un indicateur pertinent de l'état de dégradation d'un ouvrage.

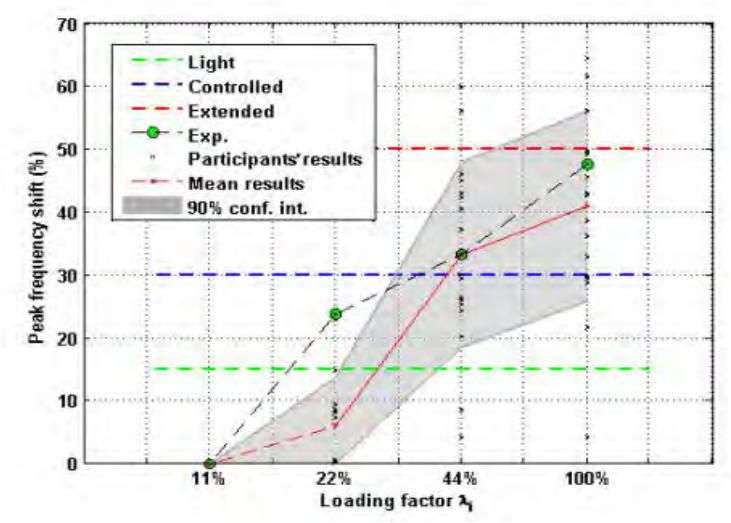

Figure 1. Evolution de la chute de fréquence du premier mode en fonction de l'augmentation du signal sismique appliqué à la maquette expérimentale (RICHARD et al. 2016).

\subsection{La solution OXAND SIMEO ${ }^{\mathrm{TM}} 4 \mathrm{D}$}

Au cours des 15 dernières années, OXAND a participé au développement de la solution SIMEO $^{\mathrm{TM}} 4 \mathrm{D}$ qui intègre une méthode d'analyse liée à l'exploitation de la réponse dynamique des ouvrages sous bruit de fond. Le système est basé sur l'utilisation de capteurs vélocimétriques communiquant entre eux par ondes radio. Cette capacité de communication permet de limiter le nombre de capteurs sur site (couramment 5 à 15 capteurs utilisés), et de produire des analyses à haut rendement en un temps d'intervention sur site réduit (en général 1 à 2 jours pour un ouvrage standard). Des illustrations de la prise des mesures sur site sont présentées figure 2 .
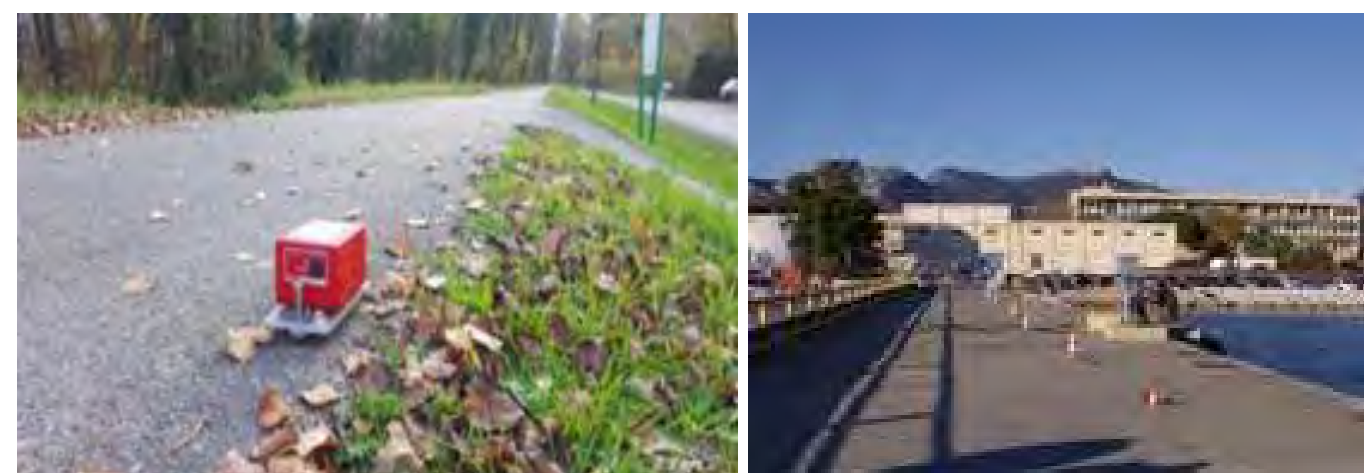

Figure 2. Prise de mesures appliquées à la caractérisation d'une digue à gauche, et lors de la cartographie des caractéristiques dynamiques d'un appontement à droite. 


\section{XIV $V^{\text {èmes }}$ Journées Nationales Génie Côtier - Génie Civil \\ Toulon, 29 juin au $1^{\text {er }}$ juillet 2016}

Le développement de cette solution industrielle repose sur les études réalisées par NAKAMURA (1989) qui a utilisé le bruit ambiant et les méthodes $\mathrm{H} / \mathrm{V}$ pour caractériser les épaisseurs des couches de sols superficiels ou les vitesses des ondes de cisaillement (Vs) des sols. Cette méthode considère que le bruit ambiant est un signal dont les caractéristiques sont proches d'un bruit blanc. La densité d'énergie spectrale est alors constante sur la plage des fréquences propres de l'ouvrage instrumenté (moyenne fréquence pour les ouvrages du génie civil, entre 0 et $50 \mathrm{~Hz}$ pour les ouvrages les plus massifs). Ce postulat a fait l'objet de plusieurs recherches richement documentées et illustrées par des campagnes expérimentales, citons par exemple BONNEFOYCLAUDET (2004) ou bien CLOTAIRE (2007). L'application de cette méthode à des ouvrages du génie civil est ancienne. IVANOVIC et al. (2000) citent une étude concernant l'estimation de la période propre d'un immeuble dès 1930. De nos jours, le développement des capteurs et la puissance des outils numériques ont amélioré les capacités de caractérisation dynamique des ouvrages : accéder à l'ensemble des modes propres sur un large spectre de fréquence $(0-200 \mathrm{~Hz})$ est aujourd'hui envisageable avec une haute précision. L'expertise de ces résultats conduit à caractériser la sensibilité de l'ouvrage à des comportements singuliers comme la torsion par exemple qui seraient des indicateurs de désordre structuraux.

Le gain apporté par cette solution d'audit est de pouvoir cartographier rapidement les caractéristiques dynamiques d'un ouvrage. A l'issue du post traitement des données, l'identification et la localisation des zones au comportement singulier (c'est-à-dire celles qui présentent une réponse différente du reste du l’ouvrage sans raison apparente) sont des informations de première importance pour caractériser l'état de l'ouvrage. Des analyses complémentaires, telles que la vérification de la capacité portante des ouvrages (voir section 3), peuvent ensuite être réalisées.

\section{Applications de l'audit structural à des ouvrages en cours d'exploitation}

L'établissement de la cartographie des caractéristiques dynamiques, ainsi que la vérification de la capacité portante d'une partie d'un appontement, ont été réalisés pour le compte de l'ESID Toulon. La cartographie de la zone instrumentée a permis de définir la position où effectuer un essai de chargement afin de vérifier la capacité portante de l'ouvrage. L'audit a été réalisé sur une durée de deux journées sur site en utilisant un jeu de 10 capteurs vélocimétriques.

\subsection{Présentation de l'ouvrage et besoins du gestionnaire}

L'ESID de Toulon exploite certains appontements (figure 3 à gauche), construits dans les années 1920. Ces ouvrages ont fait l'objet de plusieurs phases de renforcement, notamment dans les années 60, afin de prendre en compte l'évolution des usages (type et nature des navires, augmentation des masses d'exploitation à prendre en compte lors 


\section{Thème 4 - Ouvrages portuaires, offshore et de plaisance}

des phases de chargement et de déchargement). Afin de maitriser le vieillissement de ses ouvrages et contrôler la disponibilité de ses appontements,, le gestionnaire a souhaité réaliser un audit structural d'une partie d'un appontement. Les objectifs de la mission étaient les suivants :

- identifier et localiser, dans la limite de la partie instrumentée, les zones au comportement singulier nécessitant un suivi particulier ;

- estimer la capacité portante de l'ouvrage, sur la base de sa réponse dynamique acquise lors d'une série de chargements contrôlés (Figure 3 à droite, les capteurs sont visibles dans l'encadré rouge).
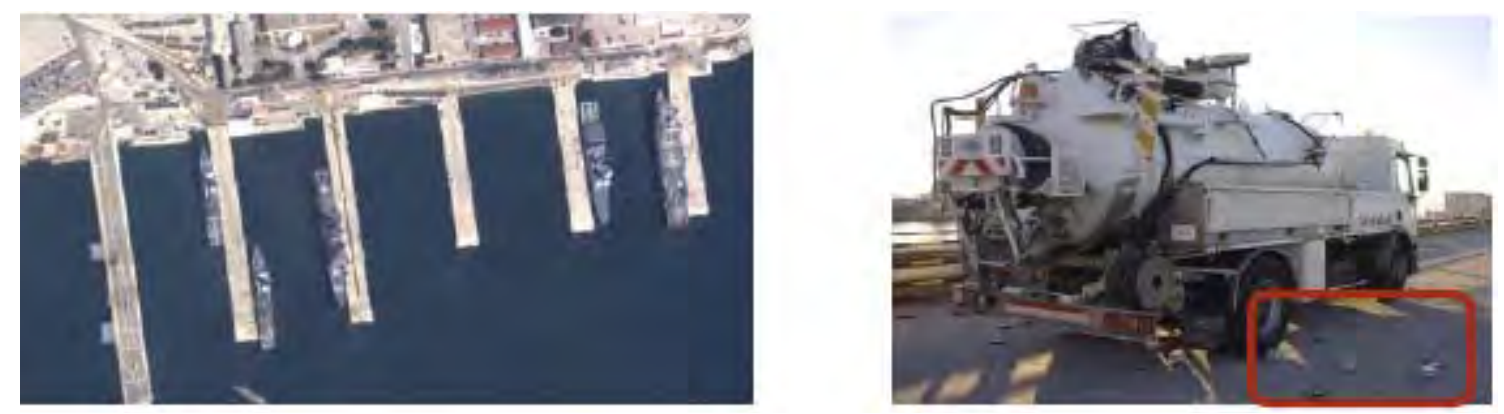

Figure 3. Vue de dessus des appontements à gauche, prise de vue depuis l'appontement lors de l'essai de chargement.

\subsection{Diagnostic à haut rendement : cartographie des caractéristiques dynamiques}

Lors de la prise des mesures sur site, 6 travées ont été instrumentées. Pour chaque travée, des mesures ont été réalisées sur les appuis et à mi- travée. Un ensemble de plus de 100 points de mesure a été réalisé dans les 3 directions. Cela équivaut à 300 spectres de réponse à analyser afin de caractériser la zone de l'appontement instrumentée. Le nombre de mesures par file dans le sens transversal (8 mesures) est justifié par la présence de prédalles de natures différentes sous la dalle de compression. La figure 4 illustre les caractéristiques mesurées en termes de fréquence et amplitude modale. Pour des raisons de confidentialité des résultats, les échelles des valeurs ne sont pas communiquées. Les couleurs chaudes (rouge) illustrent un gain en fréquence tandis que les couleurs froides (bleu) mettent un avant une perte de fréquence par rapport à la fréquence moyenne. Deux zones au comportement singulier ont été identifiées, cf. cadres rouges sur la figure 4. La zone I met en évidence une baisse de la fréquence propre (inférieure à 10\%), qui est représentative d'une perte de raideur pouvant s'expliquer par une hétérogénéité des conditions d’appuis ou une perte de rigidité des matériaux due au vieillissement. La zone II est en revanche directement impactée par la présence au cours des essais de plusieurs palettes dont le poids était proche de 4 tonnes. Cet apport de masse supplémentaire modifie localement les résultats des mesures en fréquence alors qu'en amplitude, il apparait une tendance accrue à la déformation sur les 


\section{XIV ${ }^{\text {èmes }}$ Journées Nationales Génie Côtier - Génie Civil \\ Toulon, 29 juin au $1^{\text {er }}$ juillet 2016}

zones à mi- travée. Cela indique une diminution de la raideur de l'ouvrage dans cette zone en particulier. A l'issue de ces mesures, le comportement de l'ouvrage ne présente pas de désordre significatif en termes de sécurité. Une vérification de la capacité portante a été réalisée au niveau de la travée considérée comme une zone singulière pouvant limiter la disponibilité de l’appontement.

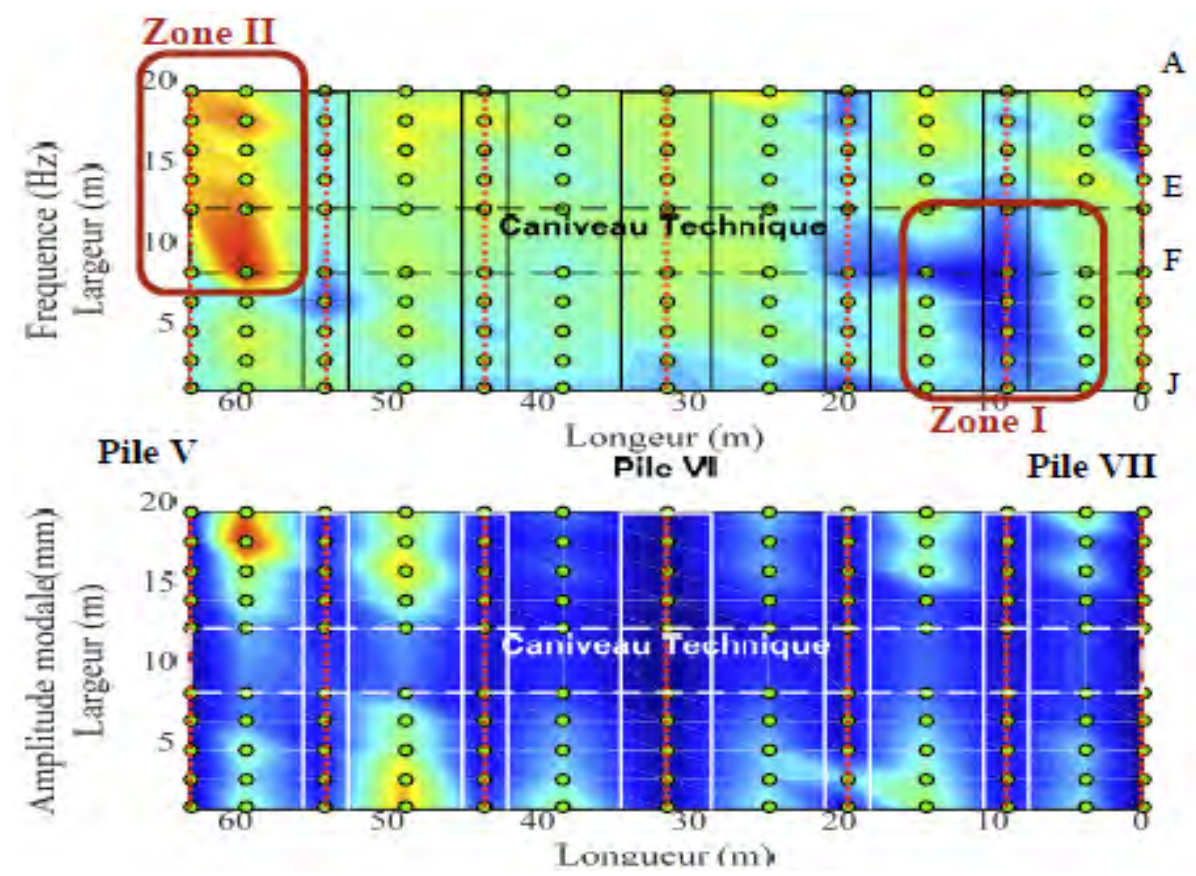

Figure 4. Cartographie des caractéristiques dynamiques de la zone instrumentée (fréquence et amplitude modale).

\subsection{Vérification de la capacité portante}

Les études réalisées par LAW \& ZHU (2005) ont mis en évidence une variation de l'état structural dès que la variation de fréquence est supérieure à $10 \%$. Ce seuil fait consensus au sein de la communauté scientifique pour caractériser le passage d'un état structural sain à dégradé. La variation de fréquence est ainsi utilisée comme indicateur pour caractériser l'état structural de l'ouvrage en phase d'exploitation, c'est-à-dire lorsque des mouvements de chargement ou déchargement ont lieu sur le quai. L'identification par analyse inverse de la masse nécessaire à une variation de la fréquence de l'ordre de 10\% est ainsi réalisée dans le but d'améliorer la disponibilité du quai par le gestionnaire.

Afin d'estimer la capacité portante de la zone de quai identifiée en section 3.2, une série de cinq chargements contrôlés a été réalisée. A chaque essai, une masse supplémentaire est ajoutée à l'aide d'un camion-citerne dont le chargement est connu afin de modifier les caractéristiques de l'appontement et, ainsi, mesurer la sensibilité de l'ouvrage à ces modifications. L'ouvrage a été mesuré à vide, puis il a été chargé à mi- travée par 
paliers successifs jusqu'à une charge nominale préalablement discutée avec le maitre d'ouvrage (capacité portante actuellement autorisée). Pour chaque mesure, la fréquence du mode vertical a été relevée. La courbe d'évolution de la fréquence montre que cette évolution reste inférieure à $10 \%$ (6\% mesurée) et illustre que la capacité portante est bien plus importante que les niveaux de charge testés. Une analyse mécanique de l’ouvrage sur la base des mesures effectuées a permis de proposer une élévation de la capacité portante de $25 \%$. Cette réévaluation de la capacité résiduelle sur la base de mesures in-situ a permis une augmentation de la disponibilité de l'appontement et donc de la performance pour l'exploitant.

\section{Conclusions}

La méthode d'audit sous bruit de fond appliquée aux ouvrages portuaires vieillissants, pour lesquels il n'existe plus ou peu d'informations relatives à leur conception, a permis de réaliser un audit structural supporté par une cartographie des caractéristiques dynamiques. Cet audit a permis d'identifier puis de localiser les zones au comportement singulier et d'évaluer la capacité portante à partir d'un essai de chargement et de calculs mécaniques. La solution $\mathrm{SIMEO}^{\mathrm{TM}} 4 \mathrm{D}$ n'impacte pas l'exploitation lors de son déploiement sur site. Non destructive, elle peut être déployée facilement et fournir rapidement des mesures à grande échelle et haut rendement d'un ouvrage (cartographie supérieure au kilomètre par jour). Ces cartographies sont utilisées non seulement pour fournir aux gestionnaires des informations concernant un état structural quantifiable de référence de leurs ouvrages, mais également pour identifier, puis localiser des zones aux comportements singuliers. Ces informations orientent les gestionnaires sur les investigations complémentaires nécessaires (inspections visuelles ou sondages destructifs) et minimisent ainsi l'impact financier sur le suivi de l'ouvrage, à court et à long terme. Appliquée à une estimation ou vérification de la capacité portante, la solution SIMEO ${ }^{\mathrm{TM}} 4 \mathrm{D}$ est une solution simple et rapide d'instrumentation qui apporte des éléments d'aide à la décision basés sur le comportement réel de l'ouvrage dans son environnement. L'analyse inverse, réalisée à partir du comportement réel sous différents chargements contrôlés, identifie la masse d'exploitation maximale permettant aux gestionnaires d'améliorer l'exploitation de ses structures tout en assurant la sécurité de l'ouvrage.

\section{Références bibliographiques}

BONNEFOY-CLAUDET S. (2004). Nature du bruit de fond sismique : implications pour les études des effets de site. Thèse de doctorat, Université Joseph-Fourier Grenoble I.

BOUTIN C., HANS S. (2009). How ambient noise measurement may help to assess building vulnerability? In: Increasing Seismic Safety by Combining Engineering 


\section{XIV ${ }^{\text {èmes }}$ Journées Nationales Génie Côtier - Génie Civil \\ Toulon, 29 juin au $1^{\text {er }}$ juillet 2016}

Technologies and Seismological Data, NATO Science for Peace and Security Series C: Environmental Security, pp 151-180.

CLOTAIRE M. (2007). Vulnérabilité sismique de l'échelle du bâtiment à celle de la ville - Apport des techniques expérimentales in situ - Application à Grenoble. Thèse de doctorat, Université Joseph-Fourier - Grenoble I.

DUNAND F. (2005). Pertinence du bruit de fond sismique pour la caractérisation dynamique et l'aide au diagnostic sismique des structures de génie civil. Thèse, Université Joseph Fourier - Grenoble I.

LAW S.S., ZHU X.Q. (2005). Nonlinear characteristics of damages concrete structures under vehicular load. Journal of Structural Engineering, ASCE, Vol. 131(8), pp 12771285. http://dx.doi.org/10.1061/(ASCE)0733-9445(2005)131:8(1277)

IVANOVIC S.S, TRIFUNAC M.D., NOVIKOVA E.I., GLADKOV A.A., TODOROVSKA M.I. (2000). Ambient vibration tests of a seven-story reinforced concrete building in Van Nuys, California, damaged by the 1994 Northridge earthquake. Soil Dynamics and Earthquake Engineering, Vol. 19, pp 391-411. http://dx.doi.org/10.1016/S0267-7261(00)00025-7

NAKAMURA Y. (1989). A method for dynamic characteristics estimation of subsurface using microtremor on the ground surface. QR of R.T.R., 30-1.

RICHARD B., CHERUBINI S., VOLDOIRE F., CHARBONNEL P.-E., CHAUDAT T., ABOURI S., BONFILS N. (2016). SMART 2013: Experimental and numerical assessment of the dynamic behavior by shaking table tests of an asymmetrical reinforced concrete structure subjected to high intensity ground motions. Engineering Structures, Vol. 109, pp 99-116. http://dx.doi.org/10.1016/j.engstruct.2015.11.029 THILLARD G., FONTAN M., RICHARD B., VOLDOIRE F., CAPRA B., MAZARS J. (2015). Benchmark SMART2013: enseignement sur les indicateurs d'endommagement pour les bâtiments en béton armé au-delà du niveau de dimensionnement. $9^{\text {ème }}$ Colloque National AFPS, Paris France, pp 1-10. 
Thème 4-Ouvrages portuaires, offshore et de plaisance 